\title{
ON THE NORMALITY OF THE PRODUCT OF TOW OPERATORS IN HILBERT SPACE
}

\section{MOHAMMED MEZIANE ${ }^{1, *}$, ABDELKADER BENALI ${ }^{2}$ AND MOHAMMED HICHEM MORTAD $^{3}$}

${ }^{1}$ Higher school of economics, B.P 65 CH2 PTT ACHABA Hnifi, Bir El Djir, Oran,Laboratoire d'analyse mathematiques et applications (LAMA), Algeria

${ }^{2}$ Faculty of The Exact sciences And computer, Mathematics Department, University of Hassiba Benbouali, Chlef Algeria. B.P. 151 Hay Essalem, chlef 02000, Algeria

${ }^{3}$ Department of Mathematics, University of Oran, B.P. 1524, El Menouar, Oran 31000, Algeria

*Corresponding author: benali4848@gmail.com

\begin{abstract}
In this paper we present the results of the maximality of operators not necessarily bounded. For that, we will see the results obtained by operators in situation of extension. Regarding the normal product of normal operators we seem to be the key to maximality.
\end{abstract}

\section{INTRODUCTION}

First, we assume that all operators operators are non necessarily bounded on a complex Hilbert space $H$, Let us, however, recall some notations that will be met below. If $A$ and $B$ are two operators with dense domains $D(A)$ and $D(B)$ respectively, then $B$ is called an extension of $A$, and we write $A \subset B$, if $D(A) \subset D(B)$ and if $A$ and $B$ coincide on $D(A)$. The product $A B$ of two operators is definded by

$$
A B(x)=A(B x) \quad \text { for } x \in D(A B)
$$

Received February 20 ${ }^{\text {th }}, 2020$; accepted April $1^{\text {st }}, 2020$; published May 19 ${ }^{\text {th }}, 2020$.

2010 Mathematics Subject Classification. Primary 47A05, Secondary 47A10, 47B20, 47B25.

Key words and phrases. normal; self-adjoint; symmetric operators; commutativity; maximality of operators.

(C)2020 Authors retain the copyrights of their papers, and all open access articles are distributed under the terms of the Creative Commons Attribution License. 
wehere

$$
D(A B)=\{x \in D(B): B x \in D(A)\}
$$

Recall too that the unbounded operator $A$, defined on $D(A)$, is said to be invertible if there exists an everywhere defined (i.e. on the whole of $\mathrm{H}$ ) bounded operator $B$, which then will be designated by $A^{-1}$, such that

$$
A^{-1} A \subset A A^{-1}=I
$$

where $I$ is the indentity operator on $H$. An operator $A$ is said to be closed if its graph is closed in $H \oplus H$. The closing of the domain $D(A)$ of $A$ implies the closing of $A$ if $A$ is bounded on $D(A)$. It is known that the product operators $A B$ is closed if for instance $A$ is closed and $B \in B(H)$, or $A^{-1} \in B(H)$ and $B$ is closed. We also recall that an operator $S$ is said to be densely defined if its domain $D(S)$ is dense in $H$. It is known that in such case its adjoint $S^{*}$ exists and is unique. If $T \subset S$, then $S^{*} \subset T^{*}$. Notice that if $S, T$ and $S T$ are all densely defined, then we are only sure of

$$
T^{*} S^{*} \subset(S T)^{*}
$$

and a full equality occurring if e.g. $T^{-1} \in B(H)$ or $S \in B(H)$. The bounded operator $T \in B(H)$ is said to be unitary if $T T^{*}=T^{*} T=I$. A densely defined operator $S$ is said to be symmetric if $S \subset S^{*}$. It is called self-adjoint if $S=S^{*}$. $S$ is called essential self-adjoint if the closure of $S$ is self-adjoint $\left(\right.$ i.e. $\left.(\bar{S})^{*}=\bar{S}\right)$. We say that $S$ is normal if $S$ is densely defined, and $\|S x\|=\left\|S^{*} x\right\|$ for all $x \in D(S)=D\left(S^{*}\right)$ (hence from known facts normal operators are automatically closed). Recall that the previous is equivalent to $S$ is closed and $S S^{*}=S^{*} S$. Other classes of operators are defined in the usual fashion. Let us also agree that any operator is linear and non necessarily bounded unless we specify that it belongs to $B(H)$. We also assume the basic theory of operators (see e.g. [1] or [20]). We do recall the celebrated Fuglede-Putnam Theorem though:

Theorem 1.1. (for a proof, see e.g. [1]) Let $T \in B(H)$ and let $M, N$ be two normal non necessarily bounded operators. Then

$$
T N \subset M T \Longrightarrow T N^{*} \subset M^{*} T
$$

One of the main objectives of this work is to impose conditions to obtain other results, starting from an extension. The following theorem and corollary result are a powerful tool to prove results on unbounded operators. For instance, Statement (3) of the next theorem is used in the proof of the "unbounded" version of the spectral theorem of normal operators (see e.g. [15]). For other uses, see e.g. [6] or [10].

Let us now list some known (see e.g. [15] or [16]) maximality results:

Theorem 1.2. Let $S, T$ be two operators with (dense when necessary) domains $D(S)$ and $D(T)$ respectively such that $S \subset T$. Then $S=T$ when one of the following occurs: 
(1) $S$ is surjective and $T$ is injective.

(2) $T$ is symmetric and $S$ is self-adjoint (resp. normal). We then say that self-adjoint (resp. normal) operators are maximally symmetric.

(3) $T$ and $S$ are normal (we say that normal operators are maximally normal). Hence, self-adjoint (resp. normal) operators are maximally normal (resp. self-adjoint).

Commutativity of operators must be handled with care. First, recall the definition of two strongly commuting (normal) operators (see e.g. [16]):

Definition 1.1. Let $A$ and $B$ be two normal operators. We say that $A$ and $B$ strongly commute if all the projections in their associated projection-valued measures commute.

Now, let us recall results obtained by Devinatz-Nussbaum (and von Neumann) on strong commutativity:

Theorem 1.3. (Devinatz-Nussbaum-von Neumann, [2] and cf. [13]). If there exists a self-adjoint operator $A$ such that $A \subseteq B C$, where $B$ and $C$ are self-adjoint, then $B$ and $C$ strongly commute.

Corollary 1.1. Let $A, B$ and $C$ be self-adjoint operators. Then

$$
A \subseteq B C \Longrightarrow A=B C
$$

\section{MAin Results}

The normality of unbounded products of normal operators has been studied recently. See e.g. [5] and the references therein. We recall

Theorem 2.1. (for a proof, see e.g. [11]) Let $A, B$ be normal operators with $B \in B(H)$. If $B A \subset A B$, then $A B$ and $\overline{B A}$ are both normal (and so $A B=\overline{B A}$ ).

Theorem 2.2. Let $T, A, B$ be non necessarily bounded operators such that $T$ and $B$ are self-adjoint with $B \in B(H)$ and $A$ is normal. Assume further that $B A \subset T$. Then

$$
\overline{B A}=T \text {. }
$$

Proof. We have:

$$
\begin{aligned}
B A \subset T & \Longrightarrow B A \subset T \subset A^{*} B \\
& \Longrightarrow B A^{*} \subset A B \text { (by Fuglede-Putnam Theorem). }
\end{aligned}
$$

It is clear that $B A$ is closable and densely defined. Let's show now that $\overline{B A}$ is normal. Indeed

$$
(\overline{B A})^{*} \overline{B A}=(B A)^{*}(B A)^{* *}=A^{*} B\left(A^{*} B\right)^{*} \supset A^{*} B B A \supset B A B A \supset B^{2} A^{*} A .
$$


Since the operators $B^{2}, A^{*} A,(\overline{B A})^{*} \overline{B A}$ are self-adjoint with $B^{2} \in B(H)$, then

$$
(\overline{B A})^{*} \overline{B A} \subset A^{*} A B^{2}
$$

by corollary 1.1 , we obtain

$$
(\overline{B A})^{*} \overline{B A}=A^{*} A B^{2}
$$

Similarly, we obtain

$$
\overline{B A}(\overline{B A})^{*}=A^{*} A B^{2},
$$

establishing the normality of $\overline{B A}$. Theorem 1.2 gives us

$$
\overline{B A}=T .
$$

Corollary 2.1. Let $T, B, A$ be non necessarily bounded operators such that $T$ is normal and $B$ symmetric and invertible (hence $B$ is self-adjoint) and that $A$ is self-adjoint, then

$$
T \subset B A \Longrightarrow A=\overline{B^{-1} T}
$$

Proof. Clearly,

$$
T \subset B A \Longrightarrow B^{-1} T \subset A,
$$

by theorem 2.2 , we obtain

$$
A=\overline{B^{-1} T}
$$

Proposition 2.1. Let $A, B$ and $T$ be operators where $B \in B(H)$. Assume that $T^{*}$ is symmetric, $B$ is self-adjoint and $A$ is normal. If $T \subset A B$, then $B A$ is essential self-adjoint.

Proof. Since $A B$ is closed, we have

$$
T \subset A B \Longrightarrow \bar{T} \subset A B,
$$

and

$$
\begin{aligned}
T \subset A B & \Longrightarrow B A^{*} \subset T^{*} \subset T^{* *}=\bar{T} \subset A B \\
& \Longrightarrow B A \subset A^{*} B \text { (by Fuglede-Putnam Theorem) } \\
& \Longrightarrow \overline{B A} \subset A^{*} B \text { (because } A^{*} B \text { is closed ). }
\end{aligned}
$$

We can show the normality of $\overline{B A}$. We have

$$
(\overline{B A})^{*} \overline{B A}=A^{*} B\left(A^{*} B\right)^{*} \supset A^{*} B B A \supset B^{2} A^{*} A .
$$


Since $B^{2}, A^{*} A$ are self-adjoint with $B^{2} \in B(H)$, then

$$
(\overline{B A})^{*} \overline{B A} \subset A^{*} A B^{2}
$$

by corollary 1.1 , we obtain

$$
(\overline{B A})^{*} \overline{B A}=A^{*} A B^{2}
$$

Similarly,

$$
\overline{B A}(\overline{B A})^{*}=A^{*} A B^{2},
$$

i.e. $\overline{B A}$ is normal. Since $(\overline{B A})^{*}$ too is normal and normal operators are maximally normal, we get

$$
\overline{B A}=(\overline{B A})^{*}=A^{*} B
$$

i.e. $B A$ is essentially self-adjoint.

Proposition 2.2. Let $T, B, A$ be non necessarily bounded operators such that $T$ is sels-adjoint and $B$ symmetric and invertible (hence $B$ is self-adjoint) and that $A^{*}$ is symmetric. Then

$$
A B \subset T \Longrightarrow T B^{-1}=\overline{B^{-1} T}=\bar{A} .
$$

Proof. Clearly,

$$
\begin{aligned}
A B \subset T & \Longrightarrow A \subset T B^{-1} \\
& \Longrightarrow B^{-1} T \subset A^{*} \subset A^{* *}=\bar{A} \subset \overline{T B^{-1}}=T B^{-1} .
\end{aligned}
$$

From theorem 2.1, we have $T B^{-1}$ and $B^{-1} T$ are normal. Hence

$$
T B^{-1}=\overline{B^{-1} T}=\bar{A} .
$$

Proposition 2.3. Let $T, B, A$ be non necessarily bounded operators such that $T$ and $B$ are self-adjoint with $B \in B(H)$ and invertible and $A^{*}$ is symmetric. If $B A \subset T$, then $B \bar{A}=\bar{A} B=T$.

Proof. We have:

$$
\begin{aligned}
B A \subset T & \Longrightarrow A \subset B^{-1} T \\
& \Longrightarrow T B^{-1} \subset A^{*} \subset A^{* *}=\bar{A} \subset \overline{B^{-1} T}=B^{-1} T .
\end{aligned}
$$

Left and right multiplying by $B$ give

$$
B T \subset B \bar{A} B \subset T B
$$

By theorem 2.1, we obtain $T B, B T$ are normal and

$$
T B=B T=B \bar{A} B
$$


hence

$$
B \bar{A}=\bar{A} B=T
$$

Theorem 2.3. Let $T, A, B$ be non necessarily bounded operators such that $A$ and $B$ are normal with $B \in$ $B(H)$ and $T$ is unitary. Assume further that $T A$ est normal. If $B A \subset T A B$ and, then $A B$ and $\overline{B A}$ are normal. Also, if $A$ and $T$ commute, then $T A B$ is normal.

Proof. Obviously,

$$
\begin{aligned}
B A \subset T A B & \Longrightarrow B^{*} A^{*} T^{*} \subset A^{*} B^{*} \\
& \Longrightarrow B^{*} T A \subset A B^{*} \text { (by Fuglede-Putnam Theorem) } \\
& \Longrightarrow B A^{*} \subset A^{*} T^{*} B
\end{aligned}
$$

It is clear that $A B$ is closed and we have:

$$
(A B)^{*} A B \supset B^{*} A^{*} A B \supset B^{*} A^{*} B A \supset B^{*} B A^{*} A .
$$

Since $(A B)^{*} A B, B^{*} B, A^{*} A$ are self-adjoint with $B^{*} B \in B(H)$, then

$$
(A B)^{*} A B \subset A^{*} A B^{*} B
$$

and by corollary 1.1, we obtain

$$
(A B)^{*} A B=A^{*} A B^{*} B .
$$

We also have,

$$
A B(A B)^{*} \supset A B B^{*} A^{*} \supset A B^{*} B A^{*} \supset B^{*} T A B A^{*} \supset B^{*} T T^{*} B A A^{*}=B^{*} B A A^{*} .
$$

Similarly, we obtain

$$
A B(A B)^{*}=A^{*} A B^{*} B \text { (because } A \text { and } B \text { are normal). }
$$

and this marks the end of the proof of the normality of $A B$. Let's show now that $\overline{B A}$ is normal. Indeed

$$
(\overline{B A})^{*} \overline{B A}=A^{*} B^{*}\left(A^{*} B^{*}\right)^{*} \supset A^{*} B^{*} B A \supset B^{*} A^{*} T^{*} B A \supset B^{*} B A^{*} A,
$$

i.e.

$$
(\overline{B A})^{*} \overline{B A}=A^{*} A B^{*} B
$$

Similarly,

$$
\overline{B A}(\overline{B A})^{*}=A^{*} A B^{*} B,
$$


that is, $\overline{B A}$ is normal.

Let's show now that $T A B$ is normal. We have $T A B$ is closed because $T$ is invertible and $A B$ is closed. we Also have

$$
\begin{aligned}
T A \subset A T & \Longrightarrow T A^{*} \subset A^{*} T \text { (by Fuglede-Putnam Theorem) } \\
& \Longrightarrow T^{*} A \subset A T^{*} \\
& \Longrightarrow T^{*} A^{*} \subset A^{*} T^{*} \text { (by Fuglede-Putnam Theorem). }
\end{aligned}
$$

Indeed

$$
T A B(T A B)^{*} \supset B^{*} A^{*} T^{*} T A B=B^{*} A^{*} A B \supset B^{*} A^{*} T^{*} B A \supset B^{*} B A^{*} A,
$$

since $T A B(T A B)^{*}, B^{*} B, A^{*} A$ are self-adjoint with $B^{*} B \in B(H)$, we get

$$
T A B(T A B)^{*} \subset A^{*} A B^{*} B
$$

By corollary 1.1, we obtain

$$
T A B(T A B)^{*}=A^{*} A B^{*} B .
$$

Similarly,

$$
(T A B)^{*} T A B=T A B(T A B)^{*}=A^{*} A B^{*} B,
$$

and this marks the end of the proof of the normality of $T A B$.

The folowing result is already seen in ( [12]), we can consider it as a consequence of the prceding theorem. Also for $T=I$ (where $I$ is the indentity operator on $H$ ) we will get the theorem 2.1 .

Corollary 2.2. Let $A, B$ be normal operators with $B \in B(H)$. Assume that $B A \subset \lambda A B$ where $\lambda \in \mathbb{C}$. Then $A B$ and $\overline{B A}$ are both normal if $|\lambda|=1$ (and so $A B=\lambda \overline{B A}$ ).

Proof. For $T=\lambda I$ where $I$ is the indentity operator on $H$, we obtain $T^{*}=\bar{\lambda} I$, i.e. $T$ is unitary (because $|\lambda|=1$ ). Theorem 2.3 yiels the normality of $A B, \overline{B A}$ and $\lambda A B$. Since $A B$ is closed, we may also write

$$
B A \subset \lambda A B \Longrightarrow \overline{B A} \subset \lambda A B
$$

But, normal operators are maximally normal, therefore, we finally infer that

$$
\overline{B A}=\lambda A B
$$

Closely related to the previous results, we have another proof for the closure of bounded operators on a domain.

Proposition 2.4. Let $T$ is a bounded operator on $D(T)$. Then $T$ is closed if $D(T)$ is closed on $H$. 
The proof requires the following lemma whose proof is very akin to the one in [15].

Lemma 2.1. Let $f: X \longrightarrow Y$ is continous such that $Y$ is Hausdorff space. Then the graph of $f$ is closed on $X \times Y$.

Now we prove proposition 2.4

Proof. We denote the graphe of $T$ by $G_{r}(T)$. By lemma 2.1, we obtain $G_{r}(T)$ is closed on $D(T) \times H$, i.e. $\left(G_{r}(T)\right)^{C}$ is open. We may write

$$
\left(G_{r}(T)\right)^{C}=\underset{(i, j) \in I \times J}{\cup} U_{i}^{1} \times U_{j}^{2}
$$

where $I, J$ are arbitrary and $U_{i}^{1}, U_{j}^{2}$ are open on $D(T), H$ respectively. Hence

$$
G_{r}(T)=\underset{(i, j) \in I \times J}{\cap}\left(F_{i}^{1} \cap D(T)\right) \times F_{j}^{2},
$$

with $F_{i}^{1}, F_{j}^{2}$ closed sets on $H$. Therefore $G_{r}(T)$ becommes closed on $H \times H$ when $D(T)$ is closed on $H$ (It's the induced topology).

Remark 2.1. In the previous proof, we did not use the linearity of $T$, we used only topological notions.

Conflicts of Interest: The author(s) declare that there are no conflicts of interest regarding the publication of this paper.

\section{REFERENCES}

[1] J. B. Conway, A course in functional analysis, (2nd edition), Springer, 1990 .

[2] A. Devinatz, A. E. Nussbaum, J. von Neumann, On the Permutability of Self-adjoint Operators, Ann. Math. 62 (2) (1955), 199-203.

[3] A. Devinatz, A. E. Nussbaum, On the Permutability of Normal Operators, Ann. Math. 65 (2) (1957), $144-152$.

[4] B. Abdelkader and H. Mortad Mohammed, Generalizations of Kaplansky's Theorem Involving Unbounded Linear Operators. Bull. Polish Acad. Sci. Math. 62 (2) (2014), 181-186.

[5] K. Gustafson, M. H. Mortad, Unbounded Products of Operators and Connections to Dirac-Type Operators, Bull. Sci. Math., 138 (5) (2014), 626-642.

[6] K. Gustafson, M. H. Mortad, Conditions Implying Commutativity of Unbounded Self-adjoint Operators and Related Topics, J. Oper. Theory, 76 (1) (2016), 159-169.

[7] Il Bong Jung, M. H. Mortad, J. Stochel, On normal products of selfadjoint operators, Kyungpook Math. J. 57 (2017), 457-471.

[8] M. H. Mortad, An All-Unbounded-Operator Version of the Fuglede-Putnam Theorem, Complex Anal. Oper. Theory, 6 (6) (2012), 1269-1273.

[9] M. H. Mortad, Commutativity of Unbounded Normal and Self-adjoint Operators and Applications, Oper. Matrices, 8 (2) (2014), 563-571.

[10] M.H. Mortad, A criterion for the normality of unbounded operators and applications to self-adjointness, Rend. Circ. Mat. Palermo, 64 (2015), 149-156. 
[11] M. Meziane, M.H. Mortad, Maximality of linear operators, Rend. Circ. Mat. Palmero, Ser. 2, 68 (2019), 441-451

[12] C. Chellali, M.H. Mortad, Commutativity up to a factor of bounded operators and applications, J. Math. Anal. Appl. 419 (2014), 114-122.

[13] A. E. Nussbaum, A Commutativity Theorem for Unbounded Operators in Hilbert Space, Trans. Amer. Math. Soc. 140 (1969), 485-491.

[14] F. C. Paliogiannis, A generalization of the Fuglede-Putnam theorem to unbounded operators, J. Oper. 2015 (2015). Art. ID 804353.

[15] W. Rudin, Functional Analysis, McGraw-Hill Book Co., Second edition, International Series in Pure and Applied Mathematics, McGraw-Hill, Inc., New York, 1991.

[16] K. Schmüdgen, Unbounded Self-adjoint Operators on Hilbert Space, Springer GTM 265 (2012).

[17] Z. Sebestyén, J. Stochel, On suboperators with codimension one domains, J. Math. Anal. Appl. 360 (2009), 391-397.

[18] J. Stochel, An asymmetric Putnam-Fuglede theorem for unbounded operators, Proc. Amer. Math. Soc. 129 (2001), 22612271.

[19] J. Stochel, F. H. Szafraniec, Domination of unbounded operators and commutativity, J. Math. Soc. Japan, 55 (2003), 405-437.

[20] J. Weidmann, Linear operators in Hilbert spaces (translated from the German by J. Szücs), Srpinger-Verlag, GTM 68 (1980). 